\title{
VALOR NUTRITIVO Y DISPONIBILIDAD DE MATERIA SECA DE PINO PONDEROSA EN SISTEMAS SILVOPASTORILES
}

\author{
Gonzalo Caballé ${ }^{1}$, Carlos Reising ${ }^{2}$ y Luis Cohen $^{1}$
}

\section{RESUMEN}

Los sistemas silvopastoriles con pino ponderosa (Pinus ponderosa) de corta edad, en los que aún no se han aplicado las primeras podas, ofrecen al alcance de los animales las ramas basales. Dependiendo de la especie animal y de la calidad y disponibilidad forrajera del pastizal, la biomasa y calidad forrajera de las acículas verdes de pino puede ser relevante en la dieta animal durante algunos años. El objetivo del presente trabajo fue evaluar la evolución de la calidad forrajera y la disponibilidad de materia seca de acículas a lo largo del periodo de veranada (diciembre-abril) en sistemas silvopastoriles en el norte de Neuquén, Argentina.

Mensualmente se tomaron muestras compuestas de acículas verdes de las ramas situadas por debajo de $1,3 \mathrm{~m}$ de altura, se secaron en estufa a $70^{\circ} \mathrm{C}$ hasta peso constante, se molieron y se obtuvieron los contenidos de lignina, proteína bruta y la digestibilidad de la materia seca. Se evaluó el contenido porcentual de fósforo total, calcio, magnesio y potasio. La disponibilidad de materia seca de acículas se estimó midiendo los diámetros de inserción al tronco de todas las ramas presentes en los verticilos por debajo de 1,3 m de altura en una muestra de árboles $(n=18)$ cubriendo el rango de diámetros a la altura del pecho (DAP, 0 a13 cm). Se extrajeron todas las acículas a una muestra de ramas $(n=54)$, se secaron en estufa $\left(70^{\circ} \mathrm{C}\right)$ hasta peso constante y luego se pesaron. Mediante regresión lineal se relacionó el peso de hojas de cada rama con el diámetro de inserción al tronco. Se estimó el peso de hojas de todas las ramas por debajo de 1,3 m y la suma total se modeló en función del DAP. A partir de la distribución de individuos por clase diamétrica se calculó la disponibilidad total de materia seca de acículas por hectárea.

El contenido de proteína bruta vario entre diciembre y marzo de 7,9 a 10,6 \%, la lignina de 13,6 a 17,5 \% y la digestibilidad de la materia seca de 52,1 a 54,7\%. El porcentaje

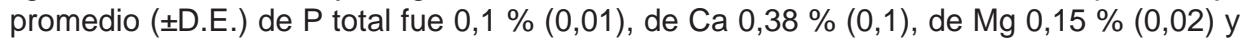
de $\mathrm{K} 0,4 \%(0,07)$. La biomasa de acículas se ajustó a un modelo potencial $\left(\mathrm{R}^{2}=0,88\right)$ con el aumento del diámetro de inserción de las ramas y la biomasa total mostró un ajuste exponencial $\left(R^{2}=0,95\right)$ con el aumento del DAP. La disponibilidad de materia seca para

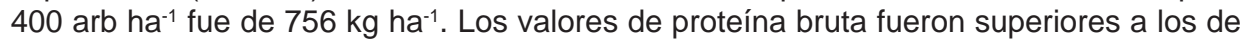
las gramíneas perennes (Poa spp., Agrostis spp., Festuca ssp, Stipa spp.) presentes en el pastizal natural en el mismo período de estudio e inferiores a los encontrados en arbustos, en su mayoría leguminosas. La digestibilidad fue muy baja, similar a la digestibilidad de la especie más pobre de las gramíneas (Stipa spp.) y los valores de macronutrientes son inferiores solo en $\mathrm{P}$ y $\mathrm{K}$ respecto a las gramíneas del pastizal. La disponibilidad estimada representa la materia seca total de un pastizal natural en buen estado de conservación y

1 INTA EEA Bariloche, CC277 (8400) Bariloche, Río Negro, Argentina. E-mail: gcaballe@bariloche.inta.gov.ar; 2 INTA Agencia de Extensión Rural, Chos Malal, Neuquén, Argentina. E-mail: creising@bariloche.inta.gov.ar 
aproximadamente el $50 \%$ de la biomasa total presente en un pastizal de borde de mallin, los sitios de mayor productividad de la Patagonia Argentina.

Los valores de proteína bruta y de disponibilidad hacen al pino una especie de valor forrajero importante en sistemas de baja productividad como los de la Patagonia Argentina. Resta evaluar el contenido de compuestos secundarios y su efecto sobre el componente animal.

Palabras clave: Sistemas silvopastorales, Pinus ponderosa 


\section{SUMMARY}

Silvopastoral systems with Ponderosa Pine (Pinus ponderosa), in which has not yet implemented the first pruning, allow the animals reach the basal branches. Depending on the animal species and the quality and availability of pasture forage, biomass and forage quality of pine needles may be relevant in animal diets for some years. The aim of this study was to assess the development of forage quality along the growing season and the availability of dry matter of needles in silvopastoral systems in northern Neuquén, Argentina.

Needles from the branches located below $1.3 \mathrm{~m}$ in height were monthly sampled, the samples were dried in an oven at $70^{\circ} \mathrm{C}$ to constant weight, ground in a mill, and lignin, crude protein content and digestibility of dry matter were analyzed. The percentage of total phosphorus, calcium, magnesium and potassium were evaluated. The availability of dry matter of needles was estimated by measuring the diameter of the trunk insertion of all branches present below $1.3 \mathrm{~m}$ in height in a sample of trees $(n=18)$ covering the range of diameters breast height $(\mathrm{DBH}, 0$ a13 cm). All needles were extracted in a sample of branches $(n=54)$, dried in an oven $\left(70^{\circ} \mathrm{C}\right)$ to constant weight and then weighted. Linear regression was fitted between leaves weight and the trunk diameter insertion. The weight of leaves of all branches below $1.3 \mathrm{~m}$ was evaluated and the total amount was modelled as a function of $\mathrm{DBH}$. Total availability per hectare of dry matter of needles was estimated from the frequency of tress by DBH classes.

The crude protein content ranged between December and March from 7.9 to 10.6 $\%$, lignin from 13.6 to $17.5 \%$ and dry matter digestibility from 52.1 to $54.7 \%$. The average $( \pm S D)$ of total P was $0.1 \%(0.01)$, Ca $0.38 \%(0.1), \mathrm{Mg} 0.15 \%(0.02)$ and $\mathrm{K} 0.4 \%(0.07)$. The biomass of needles showed a potential fit $\left(R^{2}=0.88\right)$ with increasing diameter of insertion of branches and total biomass fitted an exponential model $\left(R^{2}=0.95\right)$ with increasing

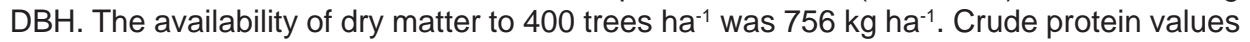
were higher than those of perennial grasses (Poa spp., Agrostis spp., Festuca spp, Stipa spp.) present in the natural grassland in the same period of study and lower than those found in legumes shrubs. The digestibility was very low, similar to the digestibility of the poorest species of grasses (Stipa spp.) and macronutrients values were lower in $\mathrm{P}$ and $\mathrm{K}$ than grasses. The estimated availability represents the total dry mater of natural grassland in good condition and approximately $50 \%$ of the total biomass present in grassland at the edge of "mallin", the higher productivity sites in Patagonia Argentina.

Because the crude protein values and availability, ponderosa pine may be an important forage species in low productivity systems such as the steppe in Patagonia Argentina. It remains to evaluate the secondary compounds and their effect on the animal component.

Key words: Agroforestry systems, Pinus ponderosa 


\section{INTRODUCCIÓN}

La Patagonia Argentina cuenta con 800 mil hectáreas de buena aptitud para el desarrollo de forestaciones con coníferas. Luego de tres décadas de promoción de la actividad desde el Estado Nacional y las Provincias se han alcanzado a forestar 82 mil hectáreas (Laclau y Andenmatten, 2005, Loguercio y Deccechis, 2006). Los resultados parecen magros. Normalmente se asigna la responsabilidad a la cultura ganadera preponderante en la región. Sin embargo, parecen ser más importantes la falta de un marco de políticas sostenidas, con fuertes altibajos en los sistemas de promoción, y los plazos productivos, muy largos propios de la actividad (Danklmaier, 2004). En este contexto, los sistemas silvopastoriles, una actividad que acelera los retornos de la inversión inicial y compatibiliza la actividad ganadera con la forestal, sin dudas puede generar un nuevo impulso a la actividad en la región.

Es particularmente interesante la implementación de sistemas silvopastoriles en el norte de la provincia de Neuquén. En esta zona, la ganadería trashumante, basada principalmente en "chiva criolla neuquina", es la principal actividad productiva. Involucra a 1.678 productores crianceros (CNA, 2002) de los cuales un tercio utilizan las tierras altas del departamento Minas como sitios de veranada. En el mismo departamento, desde mediados de la década del 60', se promueve la actividad forestal como alternativa para revertir un fuerte proceso migratorio del campo a los centros poblados. Con apoyo del Estado Nacional, pero fuertemente promocionada por el Estado Provincial y algunos municipios, desde mediado de los 70' hasta la actualidad, se lograron concretar 12.890 ha de forestaciones con especies de rápido crecimiento, siendo el pino ponderosa (Pinus ponderosa) la especie más empleada.

Sin embargo, la adopción de la forestación por parte de pequeños productores, crianceros en su mayoría, fue muy baja o prácticamente nula. La ausencia de una propuesta integradora con las otras actividades productivas que forman parte de la economía y sustento de los crianceros ha sido una de las causas. La integración de ambas actividades productivas en la misma superficie, y no el reemplazo de una por la otra, mediante la implementación de sistemas silvopastoriles, potencialmente podría aportar soluciones y compatibilizar la actividad ganadera tradicional con la actividad forestal.

Desde el punto de vista económico-productivo, en plantaciones de pino ponderosa (Pinus ponderosa) se recomienda aplicar la primera poda entre los 8 y 12 años de edad dependiendo de la calidad de sitio (Fernández et al., 2007). La práctica silvopastoril en estas plantaciones implica la introducción del ganado en el momento en que los árboles no sufran daño severo por ramoneo o vuelco. Desde que los animales se introducen en el sistema hasta la edad de la primera poda, un plazo que empíricamente puede ser de 2 a 5 años, las acículas de las ramas basales se encuentran a disposición de los animales pudiendo ser una fuente forrajera de interés dependiendo de su disponibilidad y valor nutritivo.

El consumo de la caída natural de acículas secas de pino ponderosa, con valores bajos de proteína bruta (5,3 a 5,9\%), numerosos compuestos secundarios y participaciones en la dieta superiores al 30 \%, han demostrado un efecto negativo en la nutrición de bovinos. 
Especialmente en la asimilación de nitrógeno, la digestibilidad y la tasa de pasaje de fluidos (Pfister et al., 1992). Simultáneamente, la misma calidad y magnitud causa problemas de preñez en bovinos, no así en ovinos y caprinos (Short et al., 1992, 1995). En el caso de caprinos bajo pastoreo libre, la participación en la dieta de $15 \%$ de acículas verdes provenientes del ramoneo de árboles jóvenes, no afectó la evolución en peso, condición corporal y estado de salud de la "chiva criolla neuquina" en relación a animales pastoreando en estepas graminosas arbustivas del NO de la Patagonia Argentina (Caballé et al., 2009). Contar con un estimador de disponibilidad de materia seca de acículas de pino y definir el valor nutritivo de la acícula verde parecen ser necesarios para un correcto ajuste de carga animal que asegure una dieta balanceada.

Los pastizales naturales de la Patagonia Argentina, fuera de las zonas de alta productividad (mallines), presentan un gradiente marcado en su producción definido principalmente por la distribución oeste-este de las precipitaciones y por la fuerte estacionalidad de las mismas (invierno-principio de primavera). La vegetación es codominada por gramíneas cespitosas y pequeños arbustos (Soriano, 1956, Ares et al., 1990). La producción primaria aérea es controlada durante el invierno por las bajas temperaturas y durante la primavera y el verano por la disponibilidad de agua (Jobbagy y Sala, 2000). Este conjunto de características provoca que los valores nutritivos de las principales especies forrajeras disminuyan marcadamente a medida que avanza la temporada de crecimiento y alcancen apenas para suplir los requerimientos de mantenimiento de las especies ganaderas más utilizadas (Somlo et al., 1985). Si el valor nutritivo de la acícula verde de pino durante la temporada de crecimiento, fuese superior al de las especies forrajeras o no disminuyera, podría ser utilizada como suplementación estratégica.

Mediante correcto manejo ganadero, cuidando que el consumo de acículas no supere el 15 o $20 \%$ de la dieta, la disponibilidad de acículas verdes de pino ponderosa en esta etapa temprana de los sistemas silvopastoriles, sería un componente forrajero de utilidad.

Si el valor nutritivo de la acícula fuera superior al de los pastos y se mantuviera a lo largo de la temporada de crecimiento, debido a su condición de perenne, sería una condición más para incorporar esta fuente de forraje al manejo de sistemas silvopastoriles jóvenes.

\section{OBJETIVO}

El objetivo del presente trabajo fue evaluar la evolución del valor nutritivo y la disponibilidad de materia seca de acículas verdes a lo largo del periodo de veranada (diciembre-abril) de la ganadería transhumante en sistemas silvopastoriles jóvenes en el norte de Neuquén, Argentina. 


\section{MATERIALES Y MÉTODOS}

\section{Sitio de Estudio}

Los muestreos se realizaron durante el verano 2008-2009 en el campo forestal Mallín Verde ( $36^{\circ} 56^{\prime}$ S y $70^{\circ} 48^{\prime}$ O) ubicado en la localidad de Las Ovejas, norte de Neuquén. Se encuentra a $1.650 \mathrm{msnm}$ en la zona de veranada de la ganadería transhumante. Cuenta con una superficie forestada de 690 ha. Las forestaciones con P. ponderosa se comenzaron a implantar en 1998, se realizaron 4 reposiciones y desde ese momento se excluyó el pastoreo.

Los valores históricos (15 años) de temperatura media anual y precipitación total anual son $10,6^{\circ} \mathrm{C}$ y $1.031 \mathrm{~mm}$, respectivamente, y el déficit hídrico se extiende entre noviembre y febrero (Dezzotti et al., 2008). El año 2008 presentó una temperatura media anual de $8,2^{\circ} \mathrm{C}$ y una precipitación total de $1.157 \mathrm{~mm}$.

Los suelos dominantes son Andisoles, cuyo material de origen es arenas y cenizas volcánicas Holocénicas. Los tipos de vegetación son la estepa herbácea (pastizal) (51,1 \% de la superficie), la pradera xerófila (pedrero) (12,3 \%), la pradera higrófila (mallín) (18,8 \%) y el matorral con Nothofagus antarctica (17,8 \%) (Dezzotti et al. 2008).

\section{Valor Nutritivo}

Mensualmente se tomaron muestras compuestas de acículas verdes de las ramas situadas por debajo de 1,3 m de altura. En laboratorio, el material proveniente de cada muestra se secó en estufa a $70^{\circ} \mathrm{C}$ hasta peso constante y luego fue molido en molino tipo Willey por tamiz de $1 \mathrm{~mm}$. Se realizaron los siguientes análisis: (1) Porcentaje de materia orgánica y ceniza en horno mufla a $550^{\circ} \mathrm{C}$; (2) Determinación de proteína bruta (PB) (N total x 6,25) siguiendo la metodología propuesta por O’Neill y Webb (1970); (3) Componentes membranarios, de acuerdo al fraccionamiento propuesto por Van Soest (1967) y (4) digestibilidad de la materia seca (DMS) calculada por la ecuación sumativa de Van Soest (1967).

Se evaluó, al final del periodo de crecimiento (abril), el contenido porcentual de fósforo total, calcio, magnesio y potasio en una muestra compuesta de acículas proveniente de ramas de cada uno de los tres verticilos inferiores de los árboles $(n=18)$ utilizados en el análisis de disponibilidad de biomasa (ver más adelante). Una muestra seca en estufa a $60^{\circ} \mathrm{C}$ hasta peso constante se molió en molino tipo Foss Ciclotec a un tamaño de $1 \mathrm{~mm}$. Luego se procedió a su extracción según (Harris, 1970).

Para el cálculo de fósforo total se calcinó el extracto en mufla a $550^{\circ} \mathrm{C}$ durante 8 horas. Las cenizas se analizaron por colorimetría según el método modificado de Ácido Ascórbico (Sparks y Bartels, 1996). Los cationes calcio, magnesio y potasio fueron determinados por espectrofotometría de absorción atómica (Carter, 1993; Westerman, 1995). 


\section{Disponibilidad de Biomasa de Pino}

La disponibilidad de materia seca de acículas verdes se estimó midiendo con calibre digital los diámetros de inserción al tronco de todas las ramas presentes en los verticilos por debajo de 1,3 m de altura en una muestra de árboles $(n=18)$ cubriendo el rango de diámetros a la altura del pecho (DAP, 0 a13 cm) presentes en la plantación.

Se utilizó como referencia 1,3 m ya que es la altura a la cual la "chiva criolla neuquina" ramonea sin necesidad de incorporarse sobre las patas traseras. En una rama, en cada uno de los 3 verticilos inferiores de los 18 árboles, se colectaron todas las acículas $(n=54)$. Las acículas se secaron en estufa $\left(70^{\circ} \mathrm{C}\right)$ hasta peso constante y luego se pesaron en balanza de precisión.

Mediante análisis de regresión se relacionó el peso de acículas de cada rama con el diámetro de inserción al tronco. Se estimó el peso de acículas de todas las ramas por debajo de 1,3 m y la suma total se modeló en función del DAP. A partir de la distribución de individuos por clase diamétrica por hectárea se calculó la disponibilidad total de materia seca de acículas por hectárea

\section{RESULTADOS}

\section{Valor Nutritivo}

Los tenores de proteína bruta mostraron una caída en el mes más seco (enero) con una recuperación del $17 \%$ hacia el final de la temporada.

La lignificación creció un 28 \% a lo largo de la temporada de estudio coincidente con la época seca. A causa del aumento de la lignificación la digestibilidad de la materia seca cae hacia el final de la temporada (Cuadro $N^{\circ} 1$ ).

\section{Cuadro $N^{\circ} 1$}

\section{EVOLUCIÓN DURANTE EL PERIODO DE VERANADA DEL VALOR NUTRITIVO (LINGINA, PROTEÍNA Y DIGESTIBILIDAD) DE LAS ACÍCULAS VERDES DE PINO PONDEROSA. VALORES OBTENIDOS DE MUESTRAS COMPUESTAS.}

\begin{tabular}{|l|c|c|c|c|}
\cline { 2 - 5 } \multicolumn{1}{c|}{} & Diciembre & Enero & Febrero & Marzo \\
\hline Lignina Detergente Ácido (LDA) & 13,6 & 15,1 & 16,3 & 17,5 \\
\hline Proteína Bruta (PB) & 9,0 & 7,9 & 9,2 & 10,6 \\
\hline Digestibilidad de la Materia Seca (DMS) & 54,7 & 53,4 & 52,8 & 52,1 \\
\hline
\end{tabular}

En promedio ( $\pm \mathrm{DE}$ ) el porcentaje de Fósforo total fue $0,1(0,01)$. Los cationes $\mathrm{Ca}$, Mg y K presentaron valores porcentuales promedio de $0,38(0,1), 0,15(0,02)$ y $0,40(0,07)$ respectivamente. La relación Ca:P, relevante para el crecimiento y la producción láctea, fue de 3,8 . 


\section{Disponibilidad de Biomasa de Pino}

La relación entre el peso seco $(\mathrm{g})$ de las acículas verdes y el diámetro de la rama en la inserción al tronco $(\mathrm{cm})$ en el rango de diámetros analizado $(1,2-7 \mathrm{~cm})$ se ajustó a un modelo potencial $\left(R^{2}=0,88 ; F(1,41)=310,6\right.$; ESE= 45.9) (Figura $\left.N^{\circ} 1\right)$.

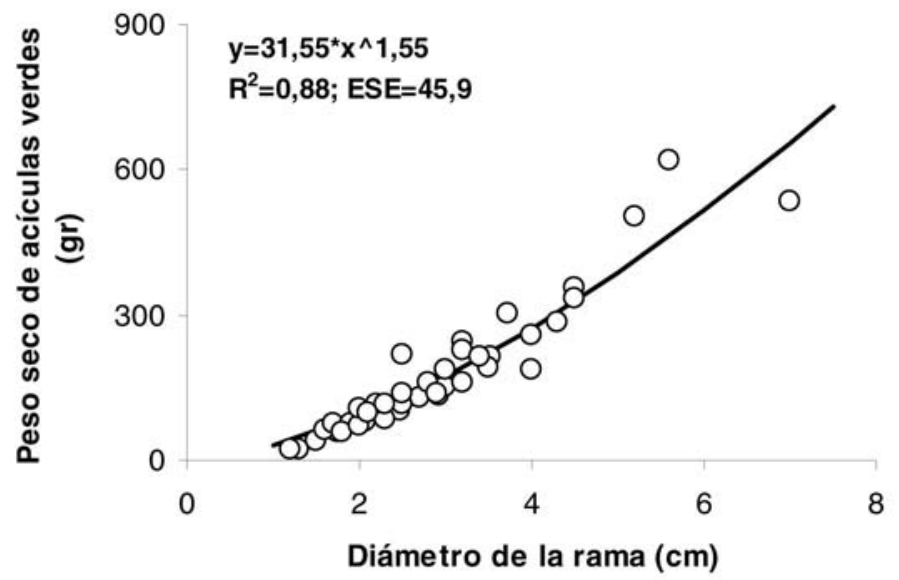

Figura $\mathrm{N}^{\circ} 1$

RELACIÓN ENTRE EL PESO SECO DE ACÍCULAS VERDES (g) Y EL DIÁMETRO DE LA RAMA EN LA INSERCIÓN AL TRONCO (cm).

Por debajo de 1,3 m de altura, el número promedio de ramas por verticilo fue 3,9. La relación entre el peso seco total $(\mathrm{kg})$ de acículas verdes por árbol (toda la biomasa presente por debajo de $1,3 \mathrm{~m})$ y su DAP $(\mathrm{cm})$ se ajustó a un modelo exponencial $\left(\mathrm{R}^{2}=0,95\right.$; $F(1,12)=307,7 ; E S E=0,3)$ (Figura $\left.N^{0} 2\right)$. 


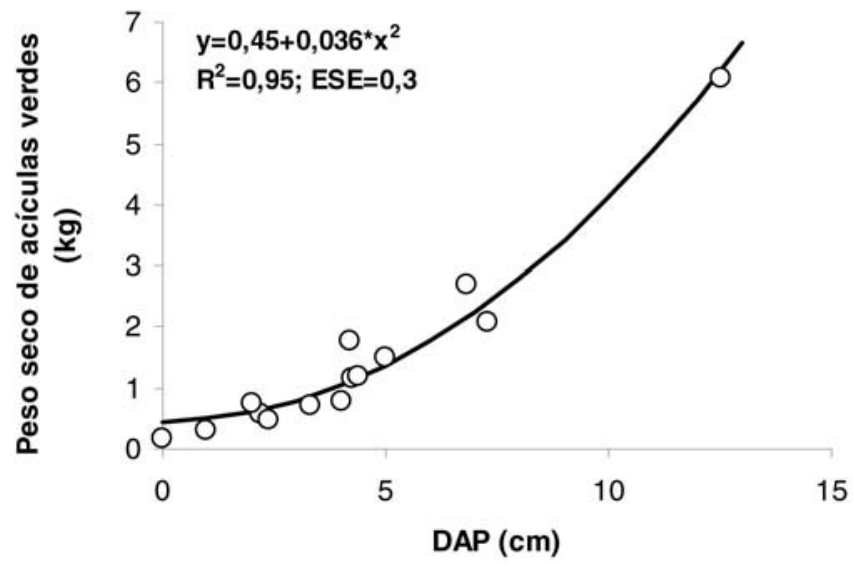

Flgura $\mathrm{N}^{\circ} 2$

RELACIÓN ENTRE EL PESO SECO DE ACíCULAS VERDES (Kg) POR DEBAJO DE 1,3 m DE ALTURA Y EL DIÁMETRO A LA ALTURA DEL PECHO (DAP) (cm).

A partir del modelo anterior se cálculo el peso seco de acículas de cada árbol "centro de clase" en la distribución de frecuencias diamétricas (clases de $2 \mathrm{~cm}$ de DAP). Multiplicando el valor obtenido con la frecuencia por clase se obtuvo la disponibilidad de materia seca por hectárea. Para el caso de estudio, 400 árb ha $^{-1}$ ponen a disposición de los animales en sus ramas basales (debajo de 1,3 m) $756 \mathrm{~kg} \mathrm{MS} \mathrm{ha}^{-1}$.

\section{DISCUSIÓN}

\section{Valor Nutritivo}

La trashumancia hacia las tierras altas de veranada comienza, dependiendo del sitio específico, entre octubre y noviembre cuando las crías tienen entre uno y dos meses. Dentro del periodo de veranada (diciembre-abril) los animales llegan con la cría al pie y se produce el destete entre enero y febrero. Los últimos meses de veranada se debe dar el crecimiento compensatorio de las madres y asegurar que la condición corporal sea suficiente para recibir el nuevo servicio ni bien se arriba a la invernada. Por este motivo, los requerimientos nutricionales considerados a continuación y sobre los que se discute la utilidad de la acícula verde de pino son un promedio de las necesidades de las crías y las madres.

El valor promedio de proteína bruta de las acículas verdes (9,2\%), a lo largo de la veranada, fue superior al de las gramíneas perennes (Poa spp., Agrostis spp., Festuca ssp, Stipa spp.; 5,7\%) presentes en el pastizal natural en el mismo período de estudio e inferiores a los encontrados en arbustos (12\%), en su mayoría leguminosas (Caballé et al., 2009). A diferencia de las gramíneas, en las que este parámetro cae marcadamente con el 
avance de la estación seca (7,2 \% a 4,6 \%), las acículas verdes del pino, exceptuando el mes de enero, mantienen los valores de proteína bruta e inclusive, al final de la temporada, lo aumentan.

No se cuenta con valores precisos de requerimiento de proteína bruta para la "chiva criolla neuquina". La raza "angora", un animal de menor tamaño, requiere luego del destete para un correcto desarrollo entre 12 y 14\% de proteína bruta y para mantenimiento entre 9 y 11\% (Huston et al.. 1971). Las diferencias encontradas entre los valores proteína de la acícula de pino y las principales especies forrajeras del pastizal, sugieren que el aporte del pino puede ser interesante, sobre todo al final de la temporada seca cuando las gramíneas tienen la mitad de proteína bruta. De todas formas, exceptuando las leguminosas, ninguna de las especies presentes cubre adecuadamente los requerimientos post-destete.

La digestibilidad de la materia seca es muy baja, similar a la digestibilidad de la especie mas pobre de las gramíneas (Stipa spp.) (Somlo et al., 1985). Esta variable se encuentra directamente relacionada con el contenido de ligninas y el aumento de la lignificación con el avance del déficit hídrico. La baja digestibilidad encontrada y los antecedentes que indican un efecto negativo de los compuestos secundarios presentes en las acículas sobre la microflora ruminal, causando indigestión (Pfister et al., 1992), seguramente jueguen en contra del aprovechamiento de las acículas por parte de los animales.

Los valores de macronutrientes son inferiores en $\mathrm{P}$ Total y $\mathrm{K}$ a valores promedio, para la misma estación de crecimiento, de las gramíneas del pastizal (Somlo et al.,1985). El valor de $\mathrm{P}$ es notablemente bajo no alcanzando a cubrir los requerimientos en lactancia $(0,22 \%$; NRC, 1968) y el de K se encuentra en el límite inferior de los requerimientos (NRC, 1989). La proporción de Ca alcanza el límite superior de los requerimientos del ganado (0,20 a 0,39\%; NRC, 1985) y se presenta en valores similares a las gramíneas del pastizal natural (Somlo et al., 1985). La utilización de Ca se ve afectada por la relación Ca:P que idealmente debería ser 2:1. En el caso de las acículas es 3,8, superior a lo ideal, pero por debajo de la relación detrimental 7:1 (NRC, 1989). El Mg se encuentra entre los valores máximos del requerimiento del ganado (NRC, 1989).

\section{Disponibilidad de Biomasa de Pino}

La relación encontrada entre el diámetro de la rama en la inserción al tronco y la biomasa de acículas en cada rama, fue similar a la encontrada por Gyenge y otros (2009) para árboles de mayor tamaño en plantaciones densas y ralas de la zona del Valle de Meliquina (4030'S, 71010'W) al sur de Neuquén. Al parecer, se trata de una relación conservadora para la especie lo cual permitiría estimar la biomasa disponible de pino a partir de la distribución diamétrica de la plantación en cualquier condición o sitio.

La disponibilidad de materia seca de acículas verdes estimada, contando con solo 400 arb ha $^{-1}$, representa la materia seca total de un pastizal natural en buen estado de conservación (Bonvissuto et al., 2008) y aproximadamente el $50 \%$ de la biomasa total presente en un pastizal de borde de "mallin" en el sitio de ensayo (Caballé et al., 2009). Los mallines son los sitios de mayor productividad de la Patagonia Argentina alcanzando en 
muy buenas condiciones 4 a 5 mil Kg MS ha-1.

\section{CONCLUSIONES}

El contenido de proteína bruta y sobre todo, el poco efecto del déficit hídrico sobre su evolución a lo largo de la veranada, confieren a la acícula verde un valor forrajero interesante. Sumado a esto, la alta disponibilidad de materia seca en relación a los pastizales donde las plantaciones se desarrollan, hacen al pino una especie de valor forrajero importante en sistemas de baja productividad como los de la Patagonia Argentina.

Si bien resta evaluar el efecto del contenido de compuestos secundarios sobre estos rumiantes menores, el pastoreo libre con ajustes precisos de carga sería posible en sistemas silvopastoriles jóvenes. De esta manera, los animales se podrían introducir antes en las forestaciones reduciendo el período de exclusión a 5-7 años.

\section{REFERENCIAS}

Ares, J., Breeskow, A. M., Bertiller, M., Rostagno, M., Irisarri, M., Anchorena, J., Defosse, G. y Merino, C.,1990. Structural and dynamic characteristics of overgrazed land of Northern Patagonia Argentina. Managed Grasslands. (Ed) Bremeyer. Amsterdam, Elsevier Science Publishers.

Bonvissuto, G. L., Somlo, R. C., Lanciotti, M. L., Gonzalez Carteau, A. y Busso, C. A., 2008. Guías de condición para pastizales naturales de "Precordillera", "Sierras y Mesetas" y "Monte Austral" de Patagonia. Ed. G. Bonvissuto, Ediciones INTA.

Caballé, G., Dezzotti, A., Sbrancia, R., Stecher, G., Reisig, C., Bonvissuto, G., Fernández, M. E., Gyenge, J. y Schlichter, T., 2009. Estudio de caso: Interacción entre el pastizal natural, la plantación de pino y el ganado caprino en el sistema silvopastoril experimental de Mallín Verde (Neuquén). Primer Congreso Nacional de Sistemas Silvopastoriles. P. Peri (Ed) Ediciones INTA, Buenos Aires, Argentina. 12 pp.

Carter, M. R., 1993. Soil Sampling and Methods of Analysis. (Ed) Martin R. Carter.

CNA, 2002. Censo Nacional Agropecuario, Instituto Nacional de Estadísticas y Censos (INDEC). Argentina.

Danklmaier, C., 2004. Análisis de los factores socioculturales que influyen la performance de los programas de promoción forestal en Chubut, Argentina". Proyectos Federales de Innovación Productiva PFIP 2004-1. Modelos de desarrollo forestal para la diversificación de los sistemas agrarios del Noroeste del Chubut. Diagnóstico social. 37 pp.

Dezzotti, A., Sbrancia, R. y Dufilho, C., 2008. Evaluación del impacto ambiental de las plantaciones de pináceas en el Campo Forestal Mallín Verde (Neuquén). Informe técnico preparado para YPF SA. $221 \mathrm{pp}$. 
Fernández, M. V., Loguercio, G. A., Ruiz Tagle-Molina, M. y Havrylenko, S., 2007. Modelo de planificación estratégica para la generación de cuencas forestales en Patagonia. Acta Primera Reunión sobre Forestación en la Patagonia, Ecoforestar 2007. Gonda, H, Davel, M, Loguercio, G y Picco, O A (Eds). Centro de Investigación y Extensión Forestal Andino Patagónico (CIEFAP), Esquel, Argentina. pp:133-144.

Gyenge, J., Fernández, M. E. and Schlichter, T. M., 2009. Effect of stand density and pruning on growth of Ponderosa Pines in NW Patagonia, Argentina. Agroforestry Systems, DOI 10.1007/s10457-009-9240-z.

Harris, L. E., 1970. Nutrition Research Techniques for Domestic and Wild Animals. Utah State University. Logan Utah. 3901 pp.

Huston, J. E., Shelton, M. y Ellis, W. C., 1971. Nutritional requirements of the Angora goat. Tex. Agr. Exp. Sta. Bull. 1105. 16 pp.

Jobbagy, E. y Sala, O., 2000. Controls of grass and shrubs aboveground production in the Patagonian steppe. Ecological Applications, 10:541-549.

Laclau, P. y Andenmatten, E., 2005. Noroeste de la Patagonia: La calidad de sitio y el negocio forestal. IDIA XXI, Forestales. Ediciones INTA, Buenos Aires, Argentina. pp: 230232.

Loguercio, G. A. y Deccechis, F., 2006. Forestaciones en la patagonia andina: potencial y desarrollo alcanzado. Patagonia Forestal, Año XII No 1. Revista editada por la Secretaría de Extensión y Divulgación del Centro de Investigación y Extensión Forestal Andino Patagónico (CIEFAP). 4 pp.

NRC. 1968. National Research Council. Nutrient requirements for domestic animals $N^{0} 5$. Nutrient requirements of sheep. National Academy of Sciences. 64 pp.

NRC. 1985. National Research Council. Ruminant N usage. National Academy Press, Washington DC, USA.

NRC. 1989. National Research Council. Nutrient requirements of Dairy Cattle, $6^{\text {th }}$ Edition update. National Academy Press, Washington DC, USA. 157 pp.

O`Nelly, J. and Webb, R., 1970. Simultaneous determination of nitrogen, phosphorus and potassium in plant material by automatic methods. Journal of the Science of Food and Agriculture, Vol. 21: 217-219.

Pfister, J. A,, Adams, D., Randall, C., Wiedmeier, D. and Cates, R. G., 1992. Adverse Effects of Pine Needles on Aspects of Digestive Performance in Cattle. Journal of Range Management, Vol. 45, No. 6:528-533.

Short, R. E,, Ford, S. P., Grings, E. E. and Kronberg, S. L.,1995. Abortifacient response and plasma vasoconstrictive activity after feeding needles from ponderosa pine trees to 
cattle and sheep. Journal of Animal Science, 73:2102-2104.

Short, R. E., James, L. F., Panter, K. E., Staigmiller, R. B., Bellows, R. A., Malcolm, J. and Ford, S. P., 1992. Effects of feeding ponderosa pine needles during pregnancy: comparative studies with bison, cattle, goats, and sheep. Journal of Animal Science, 70:3498-3504.

Somlo, R., Durañona, C. y Ortiz, R., 1985. Valor nutritivo de especies forrajeras patagónicas. Revista Argentina de Producción Animal. Vol 5 No 9-10: 588-603.

Soriano, A., 1956. Aspectos ecológicos y pastoriles de la vegetación patagónica relacionados con su estado y capacidad de recuperación. Revista de Investigaciones Agrícolas, 10: 349-379.

Sparks, D. L. y Bartels, J. M., 1996. Methods of soil analysis: Chemical Methods. Part. 3.

Van Soest, P. J., 1967. Development of a comprehensive system of feed analysis and its application to forage. Journal of Animal Science, 26: 119.

Westerman, R. L., 1995. Soil Testing and Plant Analysis, Third Edition. (Ed.) R. L. Westerman. 
